\title{
Managing cardiovascular risk in patients with diabetes
}

\author{
Lars Rydén
}

The prevalence of diabetes is increasing dramatically. If the number of patients in the developed and developing worlds with diabetes is combined, there are currently 100 million. It is predicted that this will increase to 200-225 million within the next 25 years. ${ }^{1}$

There are a number of reasons for this dramatic increase. The aging of the general population is a significant factor. Also, lack of physical exercise combined with an unhealthy diet plays an important role. As developing countries continue to adopt the bad habits of the West, the incidence of diabetes will rise.

About $90 \%$ of diabetic patients will have type 2 diabetes, which develops with age and has a strong relation to macrovascular complications.

\section{Patient outcome}

It is interesting to observe the changing patterns of mortality and morbidity in diabetic and non-diabetic patients. For non-diabetic patients there has been a successive decrease in mortality caused by cardiovascular events. ${ }^{2}$ However, in the diabetic population this has not been the case. For male diabetic patients there has been no significant change. In female diabetic patients the outcome has worsened over the years.

One reason for the poor outcome for diabetic patients is probably the divided care they receive. They are generally cared for by endocrinologists, although the main manifestations of their disease are cardiovascular which are treated by cardiologists. It is therefore important for endocrinologists and cardiologists to meet to plan patients' treatment protocols.

The risk of cardiovascular disease in the dia-

Karolinska Hospital, Department of Cardiology, Thoracic Clinic 5 tr, S-171 76 Stockholm, Sweden

Correspondence to: Professor Rydén email: lars.ryden@ks.se

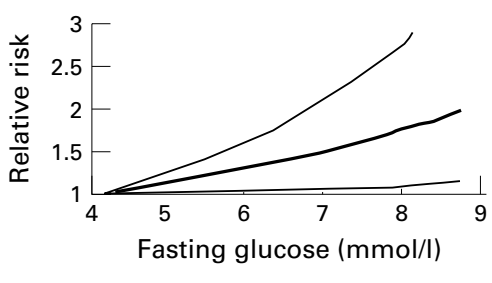

Fasting glucose of $6.1 \mathrm{mmol} / \mathrm{l}$ Postprandial glucose of $7.8 \mathrm{mmol} / \mathrm{l}$ betic population is high. For example, the MRFIT trial showed that increasing blood pressure or cholesterol concentrations increases the risk for cardiovascular events in both diabetic and non-diabetic patients. ${ }^{3}$ However, at each point the risk is much greater for diabetic patients.

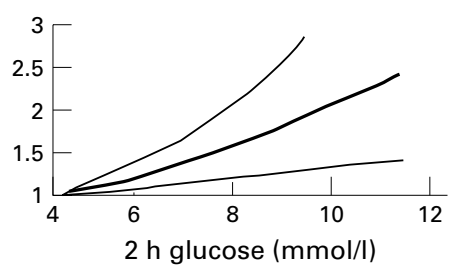

$\mathrm{RR}=1.33$ (1.06 to 1.67$)$

$\mathrm{RR}=1.58(1.19$ to 2.10$)$
Figure 1 Results of a meta-analysis looking at glucose as a risk factor for cardiovascular morbidity and mortality. Reproduced with permission from Coutinho et al. ${ }^{4}$ Copyright $(\mathrm{C}$ 1999 American Diabetes Association.

\section{Risk factors in diabetic patients}

In non-insulin dependent diabetic patients the conventional risk factors for cardiovascular disease are common. For example, approximately $70 \%$ of patients will have hypertension. However, these cannot fully explain the high mortality rates. There must be other risk factors involved.

\section{GLUCOSE}

Glucose may be an independent risk factor for cardiovascular disease. A recent meta-analysis of almost 96000 patients in 20 studies, with a follow up of 12.4 years and fasting and postprandial blood glucose measurements, ${ }^{4}$ found that there was a successive and continuous increase in the relative risk of cardiovascular mortality with increasing blood glucose concentrations (fig 1). This was true at even low concentrations of blood glucose. A fasting blood glucose of $6.1 \mathrm{mmol} / 1$, which represents borderline diabetes, resulted in a $33 \%$ higher risk for subsequent cardiovascular morbidity and mortality. With a postprandial blood glucose concentration of $7.8 \mathrm{mmol} / \mathrm{l}$, the risk is increased by $58 \%$.

This is supported by a recent study that looked at the risk of death in diabetic and nondiabetic patients with and without previous myocardial infarction (MI). ${ }^{5}$ The patients were age and sex matched and followed for eight years. The worst outcome was for patients with diabetes and a previous MI.

Interestingly, the outcome for a diabetic patient without a previous MI was the same as that for a non-diabetic patient with MI. No one would doubt the need for a patient who has had an MI to have an aggressive secondary prevention programme, but it is equally important that the diabetic patient, even without a previous MI, receives adequate treatment.

The findings of this study are confirmed by unpublished data from the OASIS group. ${ }^{6}$ This group compared diabetic and non-diabetic patients with and without unstable coronary artery disease (CAD). As expected the highest mortality was in the diabetic patients with unstable CAD. The lowest mortality was in the non-diabetic without previous manifestations of CAD. Diabetics with unstable CAD had the same outcome as non-diabetic patients without evidence of unstable CAD.

\section{BLOOD PRESSURE}

In type 1 diabetes, hypertension is usually related to nephropathy. In type 2 diabetes, however, hypertension is part of the metabolic syndrome. Therefore the vast majority of patients with type 2 diabetes have hypertension which increases the risk of cardiovascular disease. 


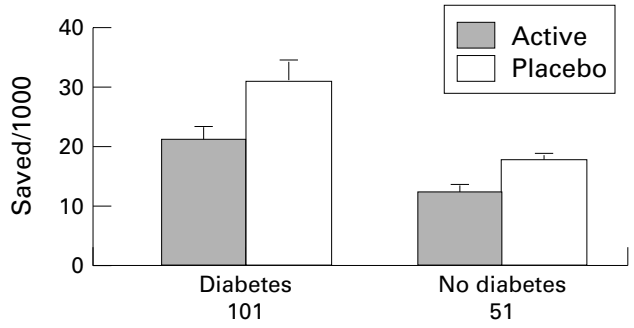

Figure 2 The efficacy of antihypertensive treatment in the diabetic patient: results from SHEP (major cardiovascular events over five years). Adapted from Curb et al. ${ }^{7}$

\section{SHEP}

One of the first studies to address the effect of antihypertensive treatment in diabetics was SHEP. ${ }^{7}$ Four thousand non-diabetic patients and 583 patients with type 2 diabetes were randomised to either the diuretic chlorthalidone with atenolol or reserpine; or placebo with any other prescription. The study found that if 1000 patients were treated, 101 diabetics would be saved from an outcome but only 51 non-diabetics would avoid a cardiac event. This demonstrates that, the higher the risk, the efficacy of treatment is increased (fig 2).

HOT

A similar study looked at the incidence of cardiovascular events in 18790 patients, 1501 of whom had diabetes. ${ }^{8}$ All patients received treatment with felodipine combined with either an angiotensin converting enzyme (ACE) inhibitor or a $\beta$ blocker. There were three target blood pressures: $\leqslant 90 \mathrm{~mm} \mathrm{Hg}$; $\leqslant 85 \mathrm{~mm} \mathrm{Hg}$; and $\leqslant 80 \mathrm{~mm} \mathrm{Hg}$. The follow up was almost four years.

In the diabetic patients there was a considerable and significant reduction in risk of major cardiovascular events with lowering of blood pressure. It is therefore important that diabetic patients are treated aggressively.

UKPDS

This finding is supported by the results of the UKPDS study. ${ }^{9}$ In this study patients with type 2 diabetes were treated with either tight or less tight blood pressure control. There was a significant $32 \%(p=0.019)$ reduction in risk of cardiovascular events in patients treated with tight blood pressure control.

UKPDS also addressed the question of whether ACE inhibitors or $\beta$ blockers have any specific advantages or disadvantages in this population. It found that compared to less well controlled blood pressure, there is actually no difference whether tight blood pressure control was achieved using an ACE inhibitor or a $\beta$ blocker.

These three studies (SHEP, HOT, and UKPDS) confirm that hypertension in the diabetic should be treated aggressively. The goal blood pressure is $\leqslant 135 \mathrm{~mm} \mathrm{Hg}$. To reach this target blood pressure a combination of drugs will usually be required.

LIPIDS

Dyslipidaemia constitutes a further important risk factor for cardiovascular disease, particu- larly in type 2 diabetes in which there is firm evidence of oxidative stress.

There is a difference in the lipid pattern between type 2 diabetics and non-diabetic subjects. Patients with type 2 diabetes are characterised by low concentrations of high density lipoprotein (HDL), a high concentration of triglycerides, and a lower rate of raised low density lipoprotein (LDL).

As cholesterol concentrations rise, there is a substantial and higher risk in the diabetic than in the non-diabetic population. ${ }^{10}$ Control of blood cholesterol and blood lipids is therefore very important in the diabetic patient.

There are three studies which provide evidence for the effectiveness of lipid lowering treatments in diabetic patients: 4S, CARE, and LIPID. ${ }^{11-13}$ However, these studies are not characteristic for the diabetic population and must therefore be interpreted with caution. For example, $4 \mathrm{~S}$ had relatively few diabetic patients, who were characterised by high blood cholesterol and not very high triglycerides which is not typical for diabetic patients. CARE and LIPID are more characteristic but not completely so.

$4 S$

In the $4 \mathrm{~S}$ study the worst outcome in terms of cardiac events was for diabetic patients who did not receive lipid lowering treatment with simvastatin. The best outcome was for nondiabetics who received treatment. Diabetics and non-diabetics who received treatment had the same prognosis. ${ }^{11}$

\section{CARE}

Diabetics had a much higher incidence of cardiac events in the CARE study. It took two or three years to achieve a significant effect in these patients with pravastatin treatment. It is therefore important that some other form of treatment is started while waiting for the beneficial effects of lipid lowering. ${ }^{12}$

\section{LIPID}

In the LIPID trial there was a $19 \%$ decrease in heart disease mortality and non-fatal myocardial infarctions in diabetics which was not significant compared to the $25 \%$ reduction in the non-diabetic group. ${ }^{13}$

A meta-analysis of the results from these three trials shows a benefit of lipid lowering in diabetic patients. However, there is a need for additional studies using more typical diabetic patients.

The new recommendation for lipid lowering from the Swedish Medical Product Agency is total cholesterol $<5 \mathrm{mmol} / \mathrm{l}$ and LDL cholesterol $<3.0 \mathrm{mmol} / 1$.

GLUCOSE

Interest has recently focused on whether the impact of blood glucose concentrations on cardiovascular risk can be reversed.

\section{UKPDS}

In the UKPDS study, intensive metabolic control using a variety of agents resulted in a $16 \%$ reduction in the number of new onset myocardial infarctions (this almost reached signifi- 


\section{Trial acronyms}

4S: Scandinavian Simvastatin Survival Study

CARE: Cholesterol and Recurrent Events

DIGAMI: Diabetes and Insulin-Glucose infusion in Acute Myocardial Infarction

HOPE: Heart Outcomes Prevention Evaluation

HOT: Hypertension Optimal Treatment

LIPID: Long-term Intervention with Pravastatin in Ischaemic Disease

MRFIT: Multiple Risk Factor Intervention Trial

OASIS: Organization to Assess Strategies for Ischemic Syndromes

SHEP: Systolic Hypertension in the Elderly Programme

UKPDS: United Kingdom Prospective Diabetes Study

cance, $\mathrm{p}=0.052) .{ }^{14}$ The reason this result was not significant may be because the patients were at relatively low risk-young, without previous disease, and at the early stage of their disease. The $16 \%$ reduction in myocardial infarction translates into the prevention of one myocardial infarction if tight metabolic control is achieved in 46 patients. This analysis can be extended to show that each per cent reduction in $\mathrm{HbA} 1 \mathrm{c}$ will result in an $18 \%$ reduction in myocardial infarction, which is very significant $(\mathrm{p}<0.0001)$.

\section{DIGAMI}

The DIGAMI study was a secondary prevention study looking at the impact of good metabolic control in post-MI diabetic patients. ${ }^{15}$ Patients were either treated rapidly with an infusion to achieve tight metabolic control or treated in any other way. Over a period of 3.4 years there was an overall reduction in mortality of $11 \%$ with good metabolic control which was significant $(\mathrm{p}=0.011)$.

HOPE

The recently completed HOPE trial provides further evidence for the need to prescribe ACE inhibitors to diabetic patients. The study included many patients with diabetes and one risk factor for cardiovascular disease who received either $10 \mathrm{mg}$ ramipril or placebo. ${ }^{16}$

Treatment with ramipril in diabetic patients reduced the primary outcome (stroke, myocardial infarction, and cardiovascular death) from $19.8 \%$ to $15.3 \%$ which was highly significant $(\mathrm{p}=0.0004) .{ }^{17}$ Moreover, in the general population of the HOPE study there was a reduction in new onset diabetes from $5.4 \%$ in the placebo group to $3.6 \%$ in the treatment group $(\mathrm{p}<0.001) .^{16}$

\section{Conclusion}

A diabetic patient is at as high a risk for a cardiac event as a patient who has suffered a myo- cardial infarction. There are a number of measures that can help these patients including antihypertensive treatment, lipid lowering treatment, good metabolic control, and also the possibility of preventing the appearance of new onset diabetes. It is therefore important that there is more widespread application of the evidence from clinical trials when treating diabetic patients.

1 Clark CM, Perry RC. Type 2 diabetes and acute macrovascular disease: epidemiology and etiology. Am Heart $\mathcal{f}$ 1999;138(5 Pt 1):330-3.

$2 \mathrm{Gu} \mathrm{K}$, Cowie CC, Harris MI. Diabetes and decline in heart disease mortality in US adults. $\mathscr{F} A M A$ 1999;281:1291-7.

3 Vaccaro O, Stamler J, Neaton JD. Sixteen-year coronary mortality in black and white men with diabetes screened for the multiple risk factor intervention trial (MRFIT). Int
f Epidemiol 1998;27:636-41.

4 Coutinho M, Gerstein HC, Wang Y, et al. The relationship between glucose and incident cardiovascular events. A metaregression analysis of published data from 20 studies of 95,783 individuals followed for 12.4 years. Diabetes Care $1999 ; 22: 233-40$

5 Haffner SM, Lehto S, Ronnemaa T, et al. Mortality from coronary heart disease in subjects with type 2 diabetes and in nondiabetic subjects with and without prior myocardial infarction. N Engl f Med. 1998;339:229-34.

6 Malmberg K, Yusuf S, Gerstein H, et al on behalf of the OASIS Registry Investigators. Impact of diabetes and longterm prognosis in patients with unstable angian and non-Q-wave myocardial infarction: results of the OASIS registry 2000. Circulation In press.

7 Curb JD, Pressel SL, Cutler JA, et al. For the systolic elderly program cooperative research group. Effect of diureticbased antihypertensive treatment on cardiovascular disease risk in older diabetic patients with isolated systolic hypertension. $7 A M A$ 1996;276:1886-92.

8 Hansson L, Zanchetti A, Carruthers SG, et al. For the HOT-study group. Effects of intensive blood pressure lowering and acetylsalicylic acid in patients with hypertension: principal results of the hypertension optimal treatment (HOT) randomised trial. Lancet 1998;351:1755-62.

9 UK Prospective Diabetes Study Group. Efficacy of atenolol and captopril in reducing the risk of macrovascular and microvascular complications in type 2 diabetes: UKPDS 39. BMF 1998;317:713-20.

10 Stamler J, Vaccaro O, Neaton JD, et al. Diabetes, other risk factors, and 12-year cardiovascular mortality for men screened in the multiple risk factor intervention trial. Diabetes Care 1993;16:434-44.

11 Pyorala K, Pedersen TR, Kjekshus J, et al. Cholesterol lowering with simvastatin improves prognosis of diabetic patients with coronary heart disease. A subgroup analysis of the Scandinavian simvastatin survival study (4S). Diabetes Care 1997;20:614-20.

12 Goldberg RB, Mellies MJ, Sacks FM, et al. Cardiovascular events and their reduction with pravastatin in diabetic and glucose-intolerant myocardial infarction survivors with average cholesterol levels: subgroup analyses in the cholesterol and recurrent events (CARE) trial. Circulation 1998;98:2513-9.

13 The Long-Term Intervention with Pravastatin in Ischaemic Disease (LIPID) Study Group. Prevention of cardiovascular events and death with pravastatin in patients with coronary heart disease and a broad range of initial cholesterol levels. N Engl F Med 1998;339:1349-57.

14 UK Prospective Diabetes Study (UKPDS) Group. Intensive blood-glucose control with sulphonylureas or insulin compared with conventional treatment and risk of complications in patients with type 2 diabetes (UKPDS 33). Lancet 1998;352:837-53.

15 Malmberg K, Norhammar A, Wedel H, et al. Glycometabolic state at admission: important risk marker of mortality in conventionally treated patients with diabetes mellitus and acute myocardial infarction. Circulation 1999;99:2626-32.

16 Heart Outcomes Prevention Evaluation Study Investigators. Effects of an angiotensin-converting enzyme inhibitor, ramipril, on cardiovascular events in high risk patients. $N$ Engl f Med 2000;342:145-53.

17 Heart Outcomes Prevention Evaluation Study Investigators. Effects of ramipril on cardiovascular and microvascular outcomes in people with diabetes mellitus: results of the HOPE study and MICRO-HOPE substudy. Lancet 2000; 355:253-9. 